\title{
Development of Online Student Assessment System: Thailand's Office of the Basic Education Commission Standardized Tests
}

\author{
Sasithorn Chutinuntakul ${ }^{1 *}$ and Prathana Phonapichat ${ }^{2}$ \\ ${ }^{l}$ Office of Registration Records and Evaluation, Sukhothai Thammathirat Open University, Thailand \\ ${ }^{2}$ School of Educational Studies, Sukhothai Thammathirat Open University, Thailand
}

\begin{abstract}
.
This study aims to develop an online student assessment system for the following subjects: Thai, Math, Science, Social Studies, Religions and Cultures, and English. The targets include students in fundamental grade 2, 4, 5, 7, 8, teachers, Educational Service Area Offices, and Office of the Basic Education Commission (OBEC). The data analysis employs Descriptive Statistics, Difficulty Index, Discriminant Index, and Reliability Index. The system developed comprises of 3 main functions: test response record, score reports, and item analysis. The response record is designed to be specifically match each subject's test patterns in that year. The score report will provide instant results in 4 level: student individual level, school level, educational service area level, and national level. Lastly, the item analysis can provide results in both individual item and test basis.
\end{abstract}

Keywords: Online Assessment; Standardized Test; Basic Education; Primary school; Secondary School

\section{Introduction}

Office of the Basic Education Commission (OBEC) provides central standardized tests for schools across the country for basic education levels including fundamental grade 2, 4, 5, 7, and 8. The tests are designed for 5 main subjects: Thai, Math, Science, Social Studies, Religions and Cultures, and English, so that to provide educational institutions in each grade with assessment results that can help develop and improve their classes and teaching processes. However, during the latest assessment, schools were required to submit the data to the central office and wait for the feedback results. This process slowed down the improvements. Some data were also incomplete, resulting in inaccurate assessment results. 
Therefore, the researchers have developed an online assessment system for the central standardized tests, focusing on the online data-collecting feature that allows schools to receive feedback results immediately after the data submission, while transferring the data to the Office of the Basic Education Commission to be used for processing the assessment in a national, area-based, regional, and provincial scale, as well as categorizing the data based on school sizes. The developed system was also designed to support data storage for use in later years.

\subsection{Objectives}

This study aims to develop an online student assessment system for the following subjects: Thai, Math, Science, Social Studies, Religions and Cultures, and English.

\subsection{Literature Review}

Presentations of the literatures that involves the development of online assessment system include information about standardized tests and assessment system development models, both of which are significant data that contribute to the developed online assessment system.

\subsubsection{Central Standardized Tests}

Data used in the development of online assessment system included the quantity of central standardized tests, categorized by grades and subjects (Tab.1), test patterns, scoring patterns, and students‘ quality levels, as follows (Office of the Basic Education Commission, 2017):

\section{Quantity of Central Standardized Tests, Categorized by Grades and Subjects}

Table 1: Quantity of central standardized tests, categorized by grades and subjects

\begin{tabular}{|c|c|c|c|}
\hline Grade & Subject & \multicolumn{2}{|c|}{ Number of Items } \\
\hline Grade 2 & Thai & \multicolumn{2}{|c|}{25} \\
\hline & & Grade 4 & Grade 5 \\
\hline \multirow[t]{3}{*}{ Grade 4-5 } & Thai & 40 & 40 \\
\hline & Math & 30 & 30 \\
\hline & Science & 30 & 30 \\
\hline & & Grade 7 & Grade 8 \\
\hline \multirow[t]{5}{*}{ Grade 7-8 } & Thai & 50 & 50 \\
\hline & Math & 30 & 30 \\
\hline & Science & 30 & 40 \\
\hline & $\begin{array}{l}\text { Social Studies, Religions and } \\
\text { Cultures }\end{array}$ & 45 & 45 \\
\hline & English & 40 & 40 \\
\hline
\end{tabular}

Source: (Office of the Basic Education Commission, 2560) 


\section{Type of Tests}

Test patterns include 5 types as follows:

1. Multiple Choices: A test that provides 4 answers for students to select the most appropriate one.

2. Complex Multiple Choices: A test that provides a main question that has multiple sub-questions within the same item that ask about different details of the main question. Each sub-question requires students to consider or assess whether it is a fact or an opinion from the given passage.

3. Multiple Responses: A test that provides multiple answers and requires students to select more than one correct answer.

4. Closed Construct/Short Answers: A test that requires students to figure out an answer from the given situation. An answer can be a solid answer or explanation regarding the set of information within a clear scope.

5. Extended-Response Questions: A test that allows students to express their opinions or thoughts that correlate, contrast or argue about the given data/situation with freedom on reasonable academic basis. This type of test needs to have clear scoring criteria.

\section{Scoring Patterns}

Scoring can be categorized into 2 main patterns: multiple choices/multiple responses where each item is assigned a fixed score; and short answers/extended-response questions where scoring for each item is scaled differently for fully correct, partly correct, and all-wrong answers.

\subsubsection{Assessment System Development Models}

Sukhothai Thammathirat Open University has developed its own test item bank that randomizes a set of items from the bank to provide computerized walk-in exams as follows:

1. Test Item Bank: This system consists of (1) Test record system that stores details about each subject such as behavioral level, purposes of the subject, difficulty value interpretation, discrimination value, and other test statistics, as well as random test standard records; (2) Test randomization system that keeps test randomization algorithms and allows for customized algorithms for randomization. The system can scope to only some sections, display available items for randomization, and report items that have never been randomized; (3) Master copymaking system that can make changes within a test, swap items, change order, record instructions in a test, and transfer into the item bank; and (4) Test analysis system that offers quality assessment for items and tests. The system can assess each individual item with classical test theory (CTT) and item response theory (IRT). It can also summarize and interpret the analysis. (Sukhothai Thammathirat Open University, n.d.)

2. Computerized Walk-In Exam System: This test management system was developed to offer computerized exams that allow students to receive instant results only as passed or failed. The system consists of test item bank management system, test management system, 
test review system, exam organizing system, test checking system, exam report system, and exam result management system. (Test Research and Development Center, Sukhothai Thammathirat Open University, 2013)

\section{Methods}

The developed online assessment system has a user-oriented design. Users include schools, Education Areas, Regional Education Offices, and the Central (OBEC). The system aims to offer accurate, fast, and cost-efficient assessment results, while support a large number of users. The assessment system was designed as follows:

1. Online system: Users at all levels can access their own results immediately without waiting for the school to send it to them.

Once the exam is finished, teachers in charge record students' answers in the system that can assess the test immediately. The results can be used to help improve class preparations and give feedback results to the students immediately. In addition to immediate results, Education Area and central offices can access the data in real-time. There is no longer need for schools to submit the data to the central office for assessment, thus greatly reducing time required compared to the conventional process.

Although users of all levels can access data in the system, the system is equipped with a security system that prevents users on the same level from accessing each other's results to ensure data continuity and accuracy.

\section{Compatibility with Changing Test Structures}

For answer input and scoring, the system was designed to allow users to select from choices shown in the system. Multiple-choice items can be input by clicking on each individual choice. In case of short-answer items, students can click and choose the value they want for the answer without having to type. This helps reduce the chance of misinput and inaccuracy that affects the assessment.

The central standardized tests are taken and assessed each year. The structure of the tests varies from one year to another. Therefore, the system was designed to allow custom configurations in terms in item quantity, test patterns, and scoring that can be tailored for each individual item. This allows OBEC officials to administrate and take responsibilities for the settings regarding the central tests by themselves.

Moreover, the researchers have also developed a test quality analysis system using the classical test theory. The platform calculates difficulty, discrimination, and KR-20 validity values, as well as displays items with desirable quality and items that need improving. This offers convenience to users as it reduces time required for laboring work such as checking the items one by one. 


\section{Thailand's Map Assessment Result Report System}

Assessment result reports can be exported into 2 formats: Excel sheet and PDF files to suit user needs. Users can also further analyze the data with the excel format. An assessment result report contains basic values: maximum, minimum, mean, standard deviation, c.v. The report classifies the student's quality into 4 levels: Very Good, Good, Fair, Poor.

Furthermore, the system was designed to report the results in the form of Thailand's geographical map with different colors representing each grade of students. Each province on the map will be marked with a distinctive color to display the overall results. This allows users to easily identify which regions or provinces need urgent attention. The map report can display results based on subjects and/or grades.

\section{Results}

The developed assessment system consists of 3 main systems: 1) Response records, 2) Item analysis, 3) Score report, as shown in fig. 1 and 2.

Figure 1: System Login

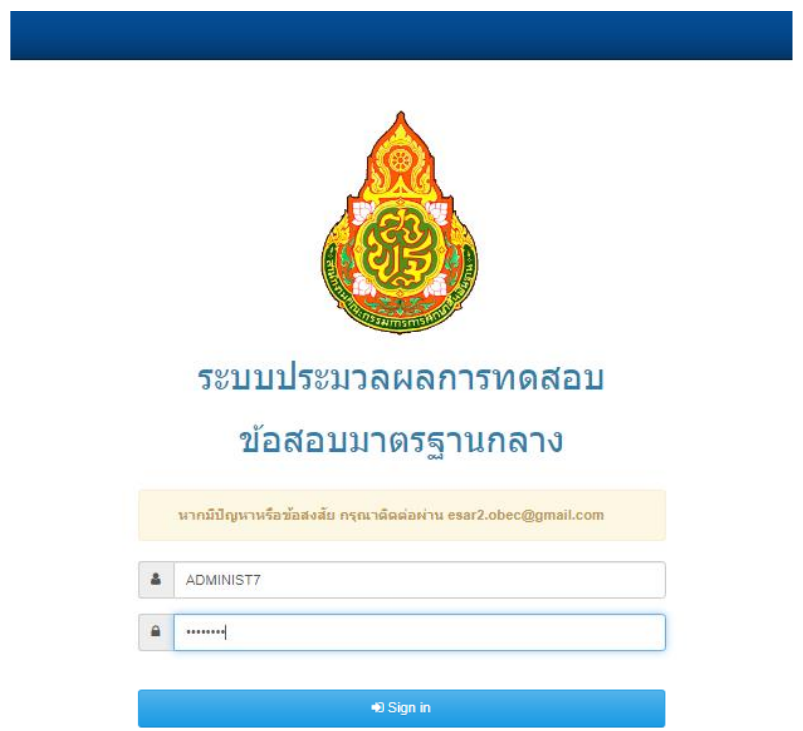

Figure 2: Menu for each system 


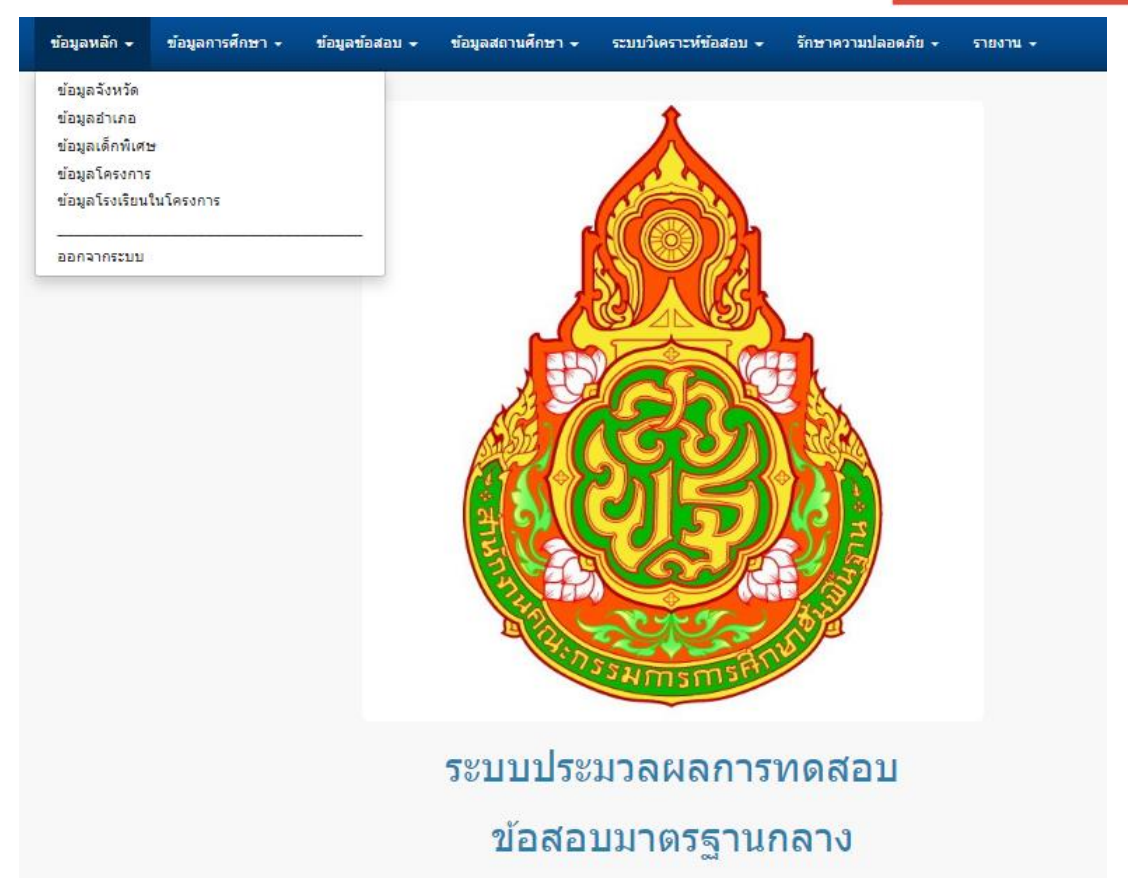

1. Response records: The records of main information such as school details, academic information, test information, and academic institution information.

Main information records contain names and codes of the provinces and regions, names and codes of students with special needs (normal, blindness, sight impairment, hearing impairment, retardation, physical disabilities, dyslexia, dyscalculia, speaking difficulties), names and codes of OBEC programs in which the school participate.

Academic Information contains details about the name and codes of subjects, standards, and indicators, as shown in fig. 3. 
Figure 3: Sample record of subject, standards, and indicators

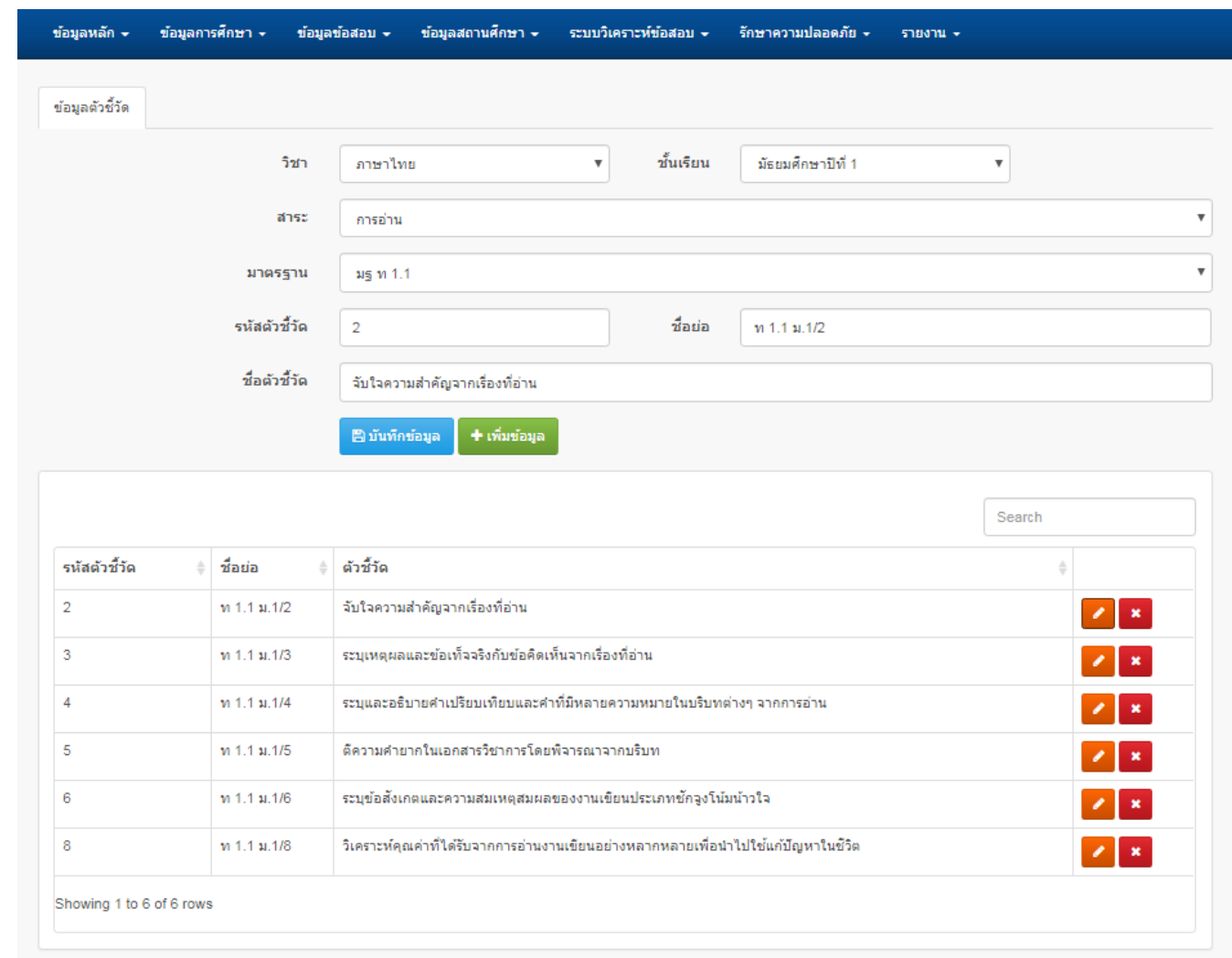

Test information contains details about: 1) Test subjects - academic year, semester $(1,2)$ subject name, quantity of items, and total score; 2) Item information - types of test, answer key, and score for each item.

Academic institution information contains details about: School details (sizes), student information (grade, class, ID number, gender, name and surname, types of special students), and student's responses to the test, as shown in fig. 4. 
Figure 4: student's responses to the test

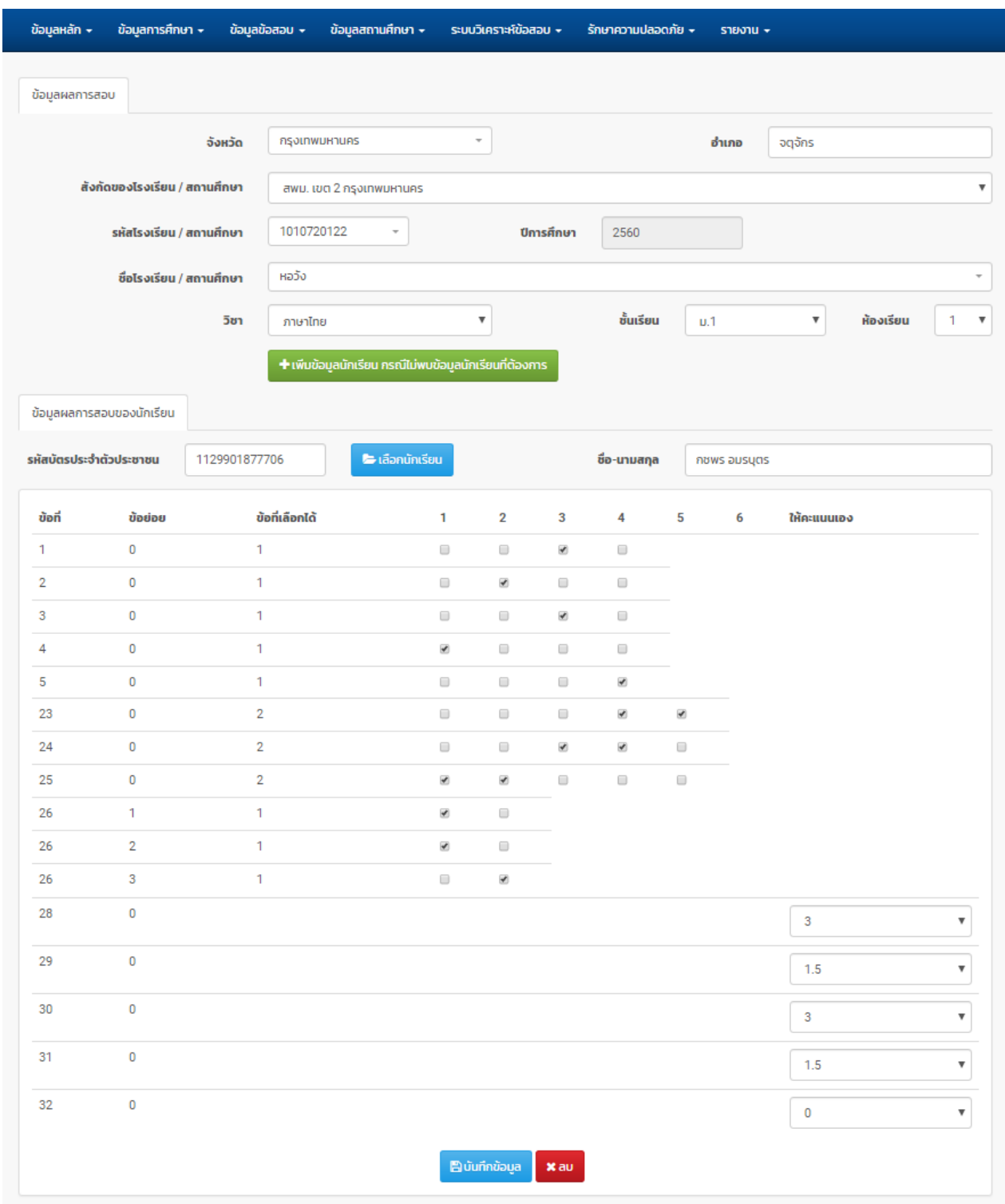

2. Analysis and Processing: This system is used for analyzing the quality of items and process the test scores. Users are required to record student's academic year/semester, subject, grade, quantity of items, total score, number of students, for the system to process the student's results.

3. Result Report: This system provides results of all levels, subjects, both in a personal and national scale. It reports overall student quality across the country in the form of Thailand's map, as well as the results of item analysis as shown in fig. 5 and 6.

- Student level: personal scores and assessment results

- School level: overall class and school results, and statistics for each subject 
- Overall national level: assessment results based on province, academic area, region, regional education office, affiliate, and school sizes

- Item analysis report: difficulty value, discrimination, validity, and quantity of itemsbased on the set criteria

Figure 5: Sample report of overall student quality in the form of Thailand's map

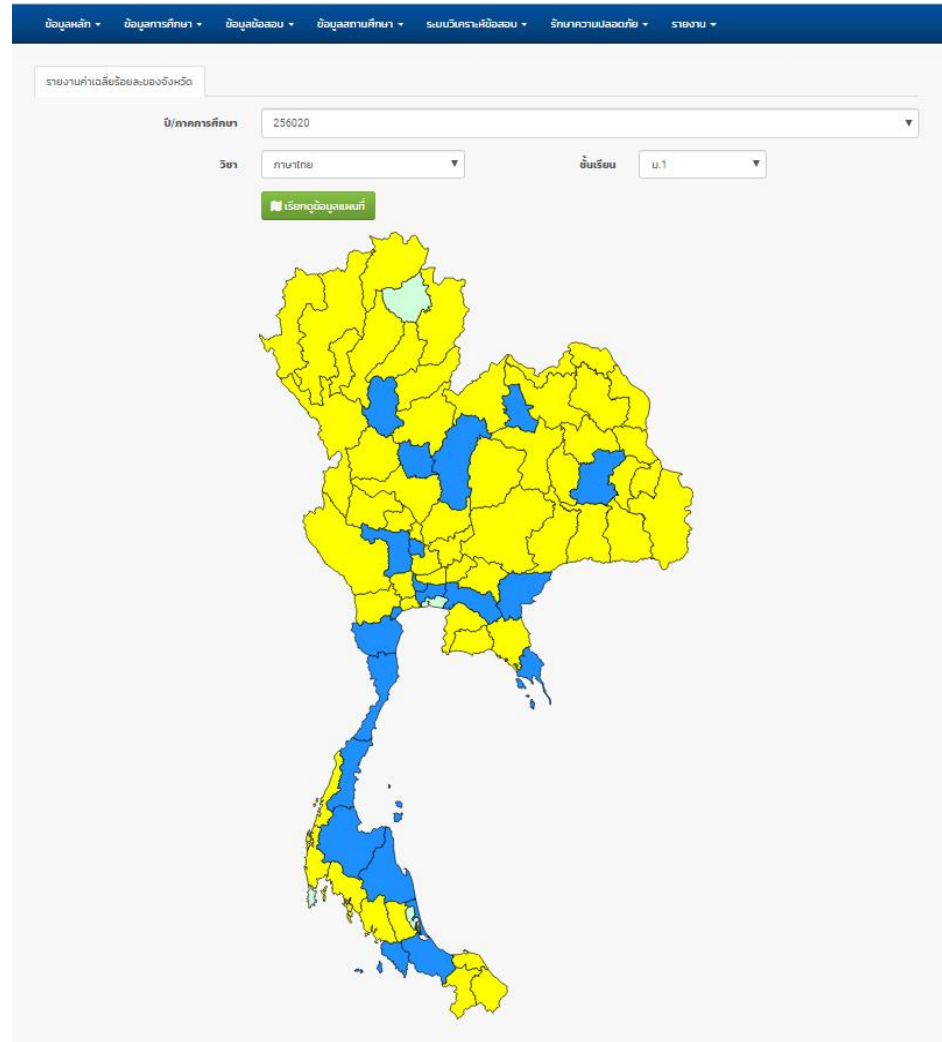

Figure 6: Sample report of Item analysis

Item Analysis Report of The Central Standardized Tests 20XX

Mathematics Grade XXX

\begin{tabular}{|c|c|c|c|c|c|c|c|c|c|c|c|c|c|}
\hline \multirow{2}{*}{ items } & \multirow{2}{*}{ keys } & \multicolumn{3}{|c|}{ choice 1} & \multicolumn{3}{|c|}{ choice 2} & \multicolumn{3}{|c|}{ choice 3} & \multicolumn{3}{|c|}{ choice 4} \\
\hline & & $p$ & $r$ & Description & $p$ & $r$ & Description & $p$ & $r$ & Description & $p$ & $r$ & Description \\
\hline 1 & & 0.47 & 0.23 & revise & 0.09 & 0.18 & $\begin{array}{c}\text { good } \\
\text { distractor }\end{array}$ & 0.06 & 0.12 & $\begin{array}{c}\text { good } \\
\text { distractor }\end{array}$ & 0.38 & 0.40 & good item \\
\hline 2 & & 0.08 & 0.17 & $\begin{array}{c}\text { good } \\
\text { distractor }\end{array}$ & 0.65 & 0.45 & good item & 0.12 & 0.23 & $\begin{array}{c}\text { good } \\
\text { distractor }\end{array}$ & 0.14 & 0.27 & $\begin{array}{c}\text { good } \\
\text { distractor }\end{array}$ \\
\hline 3 & & 0.16 & 0.25 & $\begin{array}{c}\text { good } \\
\text { distractor }\end{array}$ & 0.30 & 0.13 & $\begin{array}{c}\text { good } \\
\text { distractor }\end{array}$ & 0.44 & 0.44 & good item & 0.10 & 0.19 & $\begin{array}{c}\text { good } \\
\text { distractor }\end{array}$ \\
\hline
\end{tabular}


Apart from the 3 aforementioned systems, the system is equipped with security system that manages and assigns username and password for schools, as shown in fig. 7.

Figure 7: Sample of username and password for schools

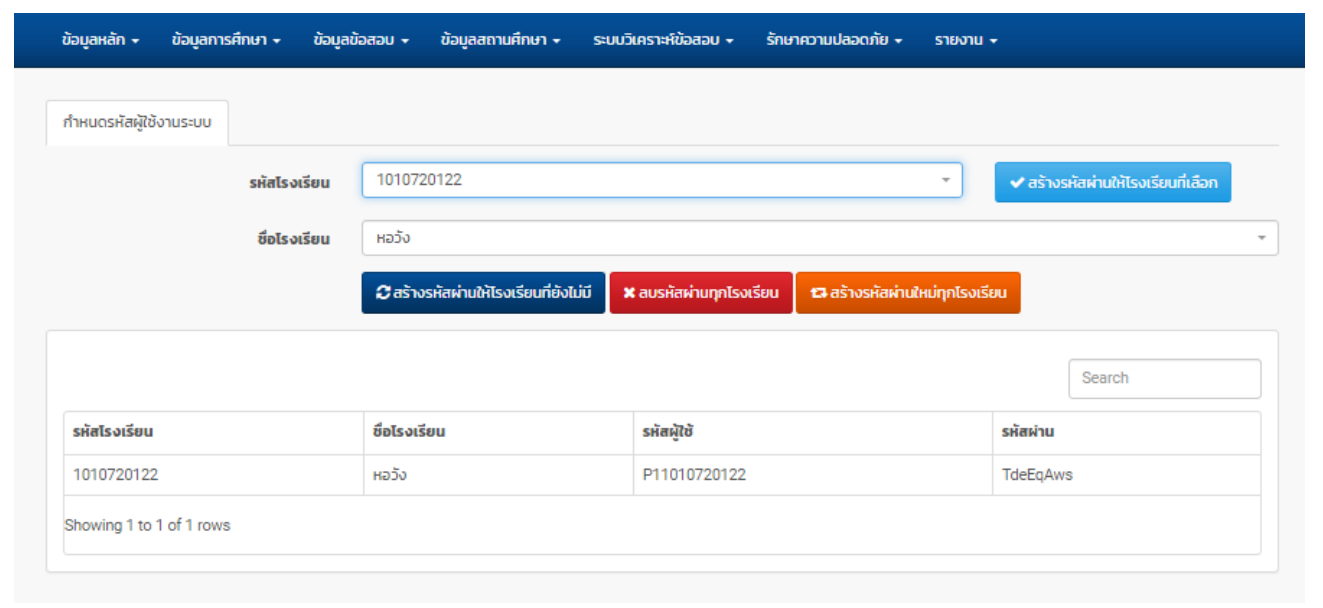

\section{Conclusion}

The developed online assessment system consists of data records of schools and students' responses, analysis and processing, assessment report, and item analysis. The system focuses on allowing users to receive the results immediately. It was specifically designed for student assessment through the central standardized tests. Due to the different structures of each test and different patterns present within each test, other freeware such as Google Form cannot completely support the tests. The reports vary based on the assessment user levels (OBEC, regional education offices, education area, school). Regarding security, users can only access their information but not other users'. Administrators can be OBEC officials themselves instead of hiring an outsource. This will save cost for allow for immediate operation.

\section{Acknowledgment}

This paper is an output of the Analysis and Processing of Assessment with Central Standardized Tests Program, funded by the Bureau of Educational Testing, Office of the Basic Education Commission, Thailand.

\section{References}

[1] Bureau of Educational Testing, Office of the Basic Education Commission. (2017). Guideline for Learning Achievement Assessment with Central Standardized Tests, 


\section{International Conference on}

\section{New Trends in Teaching and Education}

5-7 September, 2019

academic year 2017. [online]. Available:

https://sites.google.com/a/chan1.go.th/nited/khxsxb-matrthan-klang

[2] Test Research and Development center, Sukhothai Thammathirat Open University. (n.d.). Guideline for Item Bank Users. Sukhothai Thammathirat Open University. Nonthaburi: Sukhothai Thammathirat Open University.

[3] Test Research and Development center, Sukhothai Thammathirat Open University. (2013). Overall Report on Experimenting Procedures. Standard Item Bank Development Program, STOU, Nonthaburi: Sukhothai Thammathirat Open University. 